\title{
Variables que afectan a la creatividad de las personas desempleadas
}

\author{
Tomás Izquierdo-Rus ${ }^{1 *}$ y Olivia López-Martínez ${ }^{2}$ \\ ${ }^{1}$ Departamento de Métodos de Investigación y Diagnóstico en Educación. Universidad de Murcia, España \\ ${ }^{2}$ Departamento de Psicología Evolutiva y de la Educación. Universidad de Murcia, España
}

\begin{abstract}
Resumen: En este artículo se analiza la relación entre el nivel de creatividad y variables que afectan al desempleo, como la edad, el género y la duración del desempleo. Se exploraron los niveles de creatividad de 140 personas desempleadas. Los resultados derivados de la aplicación de los diferentes análisis estadísticos, utilizando una metodología cuantitativa de carácter descriptivo, nos indican que las variables estudiadas no tienen una incidencia significativa en la creatividad de las personas desempleadas. Se discute la influencia que tienen las variables mediadoras en el estudio de la creatividad de las personas que no tienen empleo. El trabajo concluye con propuestas de intervención y futuras investigaciones en el área.

Palabras clave: desempleo; creatividad; variables mediadoras; inserción la-
\end{abstract} boral.

\section{Introducción}

La Organización Internacional del Trabajo (OIT, 2002) estima que unas mil millones de personas, aproximadamente el $30 \%$ de la fuerza de trabajo total, están desempleadas o subempleadas, tanto en los países industrializados como en los países en desarrollo. Esta organización califica la situación de "sombría" y advierte que existe el riesgo de que el gran aumento numérico de los denominados "trabajadores pobres", agrave los problemas sociales y económicos provocados por las altas tasas de desempleo. España, con el $22.85 \%$ de desempleo (EPA, 2011) y una recuperación que se percibe lenta, está experimentando una transformación en muchas áreas del mercado laboral. Muchos obreros de la construcción, que ha sido el motor del crecimiento durante años, han migrado a ocupaciones del sector servicios, en particular a centros geriátricos.

Según el Servicio Público de Empleo Estatal (SPEE, 2012), los trabajos con más demanda tienen que ver con ventas, turismo, enseñanza de idiomas, negocios en internet y medio ambiente. Estos son algunos de los oficios y negocios creativos que da la crisis. España opta por la creatividad para enfrentar la crisis económica. La tasa de desempleo no sería tan alta si la población desarrollara su capacidad creativa y la pusiera en práctica, es decir, la falta de empleo, las escasas oportunidades, son fenómenos que sólo se reducen a una causa, la falta de creatividad.

Pero ¿qué entendemos por creatividad? es la capacidad de crear, de producir ideas, cosas nuevas y valiosas, es la capacidad de un cerebro para llegar a conclusiones nuevas y resolver problemas en una forma original (López, 2001). La actividad creativa debe ser intencionada y apuntar a un objetivo. En su materialización puede adoptar, entre otras, forma artística, literaria o científica, si bien, no es privativa de

* Dirección para correspondencia [Correspondence address]: Tomás Izquierdo Rus. Departamento de Métodos de Investigación y Diagnóstico en Educación. Universidad de Murcia, Campus Universitario de Espinardo s/n. 30100 Murcia (España).E-mail: tomasizq@um.es
Title: Variables affecting the creativity of people unemployed.

Abstract: In this article is evaluating the relationship between the level of creativity and variables which affect unemployment such as age, gender and period of unemployment. The creativity were explored using a sample consisted on 140 unemployed people. The results derived by application of different statistical analysis, using quantitative methodology of descriptive character, indicated that the studied mediating variables do not show any significant incidence in the creativity of people unemployed. The influence of mediating variables in the creativity of the unemployed is discussed. The work concludes with proposals for intervention and future research in the area.

Key words: unemployment; creativity; mediating variables; job placement.

ningún área en particular. La creatividad es el principio básico para el mejoramiento de la inteligencia personal y del progreso de la sociedad y es también, una de las estrategias fundamentales de la evolución natural. Es un proceso que se desarrolla en el tiempo y que se caracteriza por la originalidad, por la adaptabilidad y por sus posibilidades de realización concreta.

Creatividad es la producción de una idea, un concepto, una creación o un descubrimiento, original, útil y que satisface tanto a su creador como a otros durante algún periodo (Csikszentmihalyi, 1998). Todos nacemos con una capacidad creativa, que luego puede ser estimulada o no. Como todas las capacidades humanas, la creatividad puede ser desarrollada y mejorada (López, 2001) y especialmente debe de ser estimulada ante determinados acontecimientos, como puede ser la situación de desempleo.

Según la OIT (2012), en los 35 países sobre los que se dispone de datos, casi el 40 por ciento de las personas que buscan trabajo llevan desempleadas más de un año, lo que significa que están muy expuestos a la desmoralización, a la pérdida de autoestima y a problemas de salud mental. Se aprecia pues, una incidencia considerable de la falta de un empleo en los distintos aspectos de la existencia humana (Izquierdo y Alonso, 2010).

Resulta evidente que los desempleados no constituyen un grupo homogéneo y que, por tanto, existen diferencias individuales en la reacción ante la falta de trabajo. Esta evidencia ha determinado, según Izquierdo (2008), que la investigación preste una atención cada vez mayor a la identificación de las variables que incrementan o disminuyen los efectos del desempleo, que tiene su reflejo tanto en las actitudes hacia el empleo como en la conducta de los desempleados en el mercado laboral.

Variables como la clase social, el género, el nivel de estudios, la edad, el apoyo social o la duración del periodo de desempleo, condicionan la experiencia psicológica del desempleo. Investigadores como García y García (2008) y Garrido-Luque (2006), consideran que estas variables tienen importantes consecuencias, determinando la actitud y la 
identidad social de las personas desempleadas. Además, en muchas ocasiones, determinan otras facetas del ser humano, como es el caso de la creatividad.

La duración del desempleo ha sido una de las variables que con más frecuencia se ha utilizado en las investigaciones sobre el desempleo. Desde los inicios de la investigación psicosocial del desempleo, en los años 30, se hizo evidente que la duración del desempleo jugó un papel trascendental en el estudio de las etapas o fases por las que pasaban los desempleados. Garrido-Luque (1996) afirma que, en general, se propone un proceso en el que, tras una primera reacción de "shock", la persona desempleada mantiene aún las expectativas de encontrar un empleo y lo busca de forma activa. Posteriormente, estas expectativas van disminuyendo, lo que va acompañado de un cierto deterioro de la autoimagen y una disminución del bienestar psicológico, que desembocarán finalmente en una aceptación resignada y apática de la situación.

Algunos estudios muestran que el deterioro psicológico es mayor a medida que aumenta el periodo en desempleo. En el estudio de Wanberg (1997), se constata que es en los desempleados de larga duración, donde se encuentra un mayor empobrecimiento de aspiraciones, de autonomía y de competencia personal, que acaba mermando, entre otras dimensiones, su creatividad.

La edad es otra de las variables que modula los efectos del desempleo. Los resultados de la mayoría de las investigaciones, han mostrado una asociación significativa entre desempleo y efectos negativos. Álvaro (1992), encontró que los desempleados de mayor edad, mostraban una peor salud mental y un mayor estado depresivo, que se manifiesta en otras facetas de su vida.

Los niveles de creatividad varían con la edad, ya que esta variable no sólo acarrea variaciones del funcionamiento individual, ya sea físico o psicológico, sino también, como afirma Banks (1992), en las responsabilidades de los roles que se asuman a lo largo del ciclo vital, que contribuyen a explicar las variaciones que se observan en las respuestas al desempleo.

Otra de las variables consideradas a la hora de estudiar las diferencias individuales ante el desempleo ha sido el género. No obstante, en la evaluación de este factor, no sería correcto recurrir a simples comparaciones entre hombres y mujeres. Esto indica que hemos de tener cuidado, a la hora de establecer comparaciones, para no generalizar resultados, sin tener en cuenta la mayor complejidad del rol laboral de las mujeres, en comparación con los hombres (GarridoFernández, 1995).

En diferentes investigaciones (Banks, 1992; Buendía, 1990; Caputo y Cianni, 2001; Garrido-Luque, 1996; Izquierdo, 2005; Izquierdo, 2008; Kulik, 2001; Sánchez, 1994), se encuentra que el impacto del desempleo sobre las mujeres es tan negativo como en los hombres. No obstante, sería necesario tener en cuenta la incidencia de otras variables mediadoras, que pudieran incidir de alguna manera en esta relación (Izquierdo, 2008) y que puedan tener una incidencia directa en los niveles de creatividad, tanto de hombres como de mujeres.

Esta investigación forma parte de un estudio más amplio cuyo propósito es estudiar la creatividad en personas desempleadas y su relación con variables personales y profesionales. Variables como la edad, el género y el tiempo en desempleo, constituyen el núcleo fundamental de este trabajo. A través de las mismas hemos tratado de conocer y analizar el nivel de creatividad de las personas en situación de desempleo.

\section{Método}

Presentado el marco teórico en el que se desenvuelve esta investigación, a continuación se aborda el trabajo empírico utilizando una metodología cuantitativa, de carácter descriptivo, con el objetivo de estudiar la incidencia de variables como la edad, el género y el tiempo en desempleo en la creatividad de las personas desempleadas. Con este fin se describen los participantes en la investigación, el instrumento de recogida de información y el análisis de los datos obtenidos.

\section{Participantes}

Participaron en la investigación 140 personas inscritas como demandantes de empleo en el Servicio Andaluz de Empleo, distribuidos en cuatro grupos en función del tiempo en desempleo: $14.4 \%$ ( $<3$ meses), $25.5 \%$ ( $>3-<12$ meses), $24.8 \%$ ( $>12-<24$ meses) y $35.3 \%$ ( $>24$ meses).

La edad media de los participantes es de 36 años, con una desviación típica de 11.35 y un rango de 17 a 62 años. Se establecieron tres grupos tomando la clasificación propuesta por el Servicio Público Empleo Estatal ( $<25$ años, 25-44 años $\mathrm{y}>45$ años). Finalmente, la distribución por género quedó compuesta por 103 mujeres $(73.6 \%$ ) y 37 hombres $(26.4 \%)$.

\section{Instrumento}

El instrumento utilizado para esta investigación consta de dos partes; en una primera parte se incluyen preguntas socioprofesionales, concretamente edad, género y tiempo en desempleo y, en segundo lugar, para medir la creatividad, se aplicó el test "CREA. Inteligencia creativa" (Corbalán, Martínez, Donolo, Tejerina y Limiñana, 2003).

El CREA es una medida cognitiva de la creatividad a través de la generación de preguntas. Utiliza como procedimiento para la medida de creatividad, la capacidad del sujeto para elaborar preguntas a partir de un material gráfico suministrado. La prueba se puede aplicar tanto individual como colectivamente, siendo el tiempo de duración necesario, entre 10 y 20 minutos. Este test proporciona una puntuación directa (PD), que debe ser consultada en los baremos correspondientes para ser interpretada. A tal fin, los autores presentan una tabla de criterios interpretativos generales, sobre las características creativas de sujetos, basados en los percentiles y agrupados, según la puntuación obtenida en la 
prueba respectiva, como creatividad alta, media o baja. Por ejemplo, en la posición alta se indica que los sujetos tienen excelentes posibilidades para desarrollar tareas de innovación y producción creativa (López y Navarro, 2008). Según estos autores, el CREA cumple los estándares básicos de fiabilidad y validez exigibles a una prueba psicológica.

\section{Análisis de datos}

El análisis de los datos se ha realizado utilizando el software estadístico PASW STATISTICS 18. Se emplearon las frecuencias y porcentajes para presentar el nivel de creatividad mostrada por los desempleados en las respuestas proporcionadas al CREA. Para el análisis de las diferencias en Creatividad respecto al género se ha utilizado la $t$ de student y para las variables edad y tiempo en desempleo, tras garantizar que cumplían los requisitos para realizar pruebas pa- ramétricas, se ha utilizado el análisis de varianza de un factor (ANOVA).

\section{Resultados}

El CREA nos permite apreciar el carácter creativo de los sujetos, en función de factores como la edad, el género y el tiempo en desempleo. Estos factores configuran, en muchos casos, una determinada actitud, que se traduce en diferentes niveles de creatividad. En duración de desempleo, al comparar los diferentes niveles de creatividad, se encuentra un mayor número de sujetos con creatividad media. Se aprecia además, un elevado número de sujetos con mayor tiempo en desempleo ( $>24$ meses) en todos los niveles (baja, media y alta). En la Tabla 1 se muestra la frecuencia obtenida por cada uno de los grupos analizados, medida a través del CREA, como indicador para interpretar el perfil creativo de los desempleados.

Tabla 1. Frecuencias en CREA en función del tiempo en desempleo.

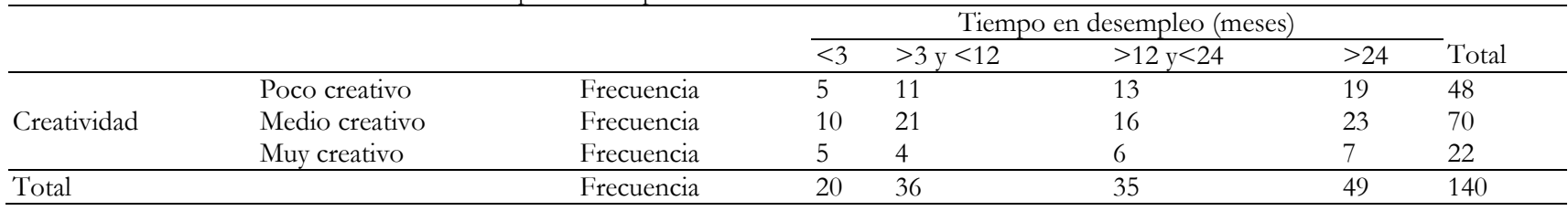

Los resultados del ANOVA no arrojan resultados significativos (Tabla 2), por lo que no podemos afirmar que existan diferencias significativas en creatividad respecto a la duración del desempleo. En muchas ocasiones los sujetos con mayor tiempo en situación de desempleo se sienten desmotivados y con poca energía para afrontar esta situación, sin embargo esto no afecta a su nivel de creatividad.

Tabla 2. Resultados de la prueba ANOVA en el test CREA.

\begin{tabular}{llllll}
\hline & $\begin{array}{l}\text { Suma de } \\
\text { cuadrados }\end{array}$ & gl & Media cuadrática & $F$ & $p$ \\
\hline Inter-grupos & .871 & 3 & .290 & .614 & .607 \\
Intra-grupos & 64.300 & 136 & .473 & & \\
Total & 65.171 & 139 & & & \\
\hline
\end{tabular}

Los valores obtenidos en los niveles de creatividad respecto al género, indican que hay una ligera tendencia de aumento, tanto hombres como mujeres, desde poca creatividad a creatividad media, siendo ésta última donde se encuentra el mayor número de personas. Esta tendencia se reduce, sin embargo, en creatividad alta con un menor número de desempleados de ambos géneros (Tabla 3).

Tabla 3. Frecuencias en CREA en función del género.

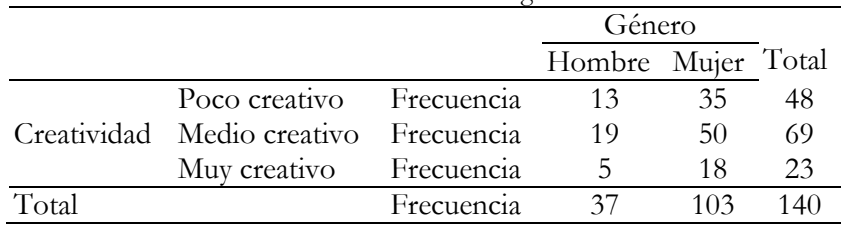

$\mathrm{Al}$ aplicar la $t$ de student no se aprecian diferencias significativas en el perfil de creatividad respecto al género (Tabla 4). La influencia de esta variable es distinta a la que puedan ejercer otras variables debido, en su mayoría, a que las consecuencias no son las mismas dependiendo del género al que pertenezcan. Conviene destacar que hay una diferencia considerable en el tamaño del género de pertenencia, debido a que es mayor la afluencia y participación de las mujeres en los Servicios Públicos de Empleo.

Tabla 4. Resultados de la prueba $t$ de student en el test CREA.

\begin{tabular}{llllll}
\hline & $F$. & $p$ & $t$ & gl & $p$ (bilateral) \\
\hline $\begin{array}{l}\text { Se han asumido } \\
\text { varianzas iguales }\end{array}$ & .049 & .825 & -.343 & 146 & .732 \\
$\begin{array}{l}\text { No se han asumido } \\
\text { varianzas iguales }\end{array}$ & & & & & \\
\hline
\end{tabular}

En referencia a la edad, se trató de establecer si hay diferencias en creatividad en función de esta variable y, de existir, si se acentuaban o no con la edad. Es en creatividad media donde se observa el mayor número de sujetos, destacando el grupo de personas con edades comprendidas entre 25 y 44 años (Tabla 5).

Tabla 5. Frecuencias en CREA en función de la edad.

\begin{tabular}{lllllll}
\hline & & & \multicolumn{5}{c}{ Edad (grupos) } \\
& & & \multicolumn{2}{c}{$<25-44$} & $>=45$ & Total \\
\hline \multirow{3}{*}{ Creatividad } & Poco creativo & Frecuencia & 7 & 29 & 12 & 48 \\
& Medio creativo & Frecuencia & 14 & 31 & 24 & 69 \\
& Muy creativo & Frecuencia & 6 & 13 & 4 & 23 \\
\hline \multirow{2}{*}{ Total } & Frecuencia & 27 & 73 & 40 & 140 \\
\hline
\end{tabular}


Los resultados obtenidos de la aplicación del análisis de varianza (Tabla 6), muestran que no existen diferencias significativas en estilos de creatividad, respecto a la edad. Esto nos indica la gran homogenidad en estilos de creatividad respecto a los grupos de edad establecidos. La situación de desempleo se asocia, en muchos casos, con una disminución del autoconcepto y de la posición social valorada, independiente de la edad, lo que ocasiona que los niveles de creatividad sean similares.

\begin{tabular}{lcclll}
\multicolumn{6}{l}{ Tabla 6. Resultados de la prueba ANOVA en el test CREA. } \\
\hline & Suma de cuadrados & gl & $\begin{array}{l}\text { Media } \\
\text { cuadrática }\end{array}$ & F & $p$ \\
\hline Inter-grupos & .466 & 2 & .233 & .485 & .617 \\
Intra-grupos & 69.609 & 145 & .480 & & \\
Total & 70.074 & 147 & & & \\
\hline
\end{tabular}

\section{Discusión}

Los resultados derivados de la aplicación de los diferentes análisis, nos indican que las variables estudiadas no tienen una incidencia significativa en la creatividad de las personas desempleadas, lo que se aprecia en las diferentes pruebas de significación realizadas. En los análisis de las variables como edad, género y tiempo en desempleo no se aprecian diferencias significativas, al obtenerse un grado de significación superior a .05 en cada una de ellas.

Como se pone de manifiesto en los resultados obtenidos, un amplio número de participantes manifiesta poca o media creatividad, como consecuencia de la situación de desempleo en la que se encuentran. A medida que el periodo en desempleo aumenta desciende el nivel de creatividad. Esto coincide con las aportaciones de Piqueras y Rodríguez (1997), que afirman que los desempleados presentan una situación de desgaste personal, que es necesario atender con metodologías específicas de intervención.

También es motivo en este estudio, comprobar si la creatividad de las personas desempleadas varía en función de la edad. Los resultados obtenidos nos indican que esta variable afecta de forma generalizada a los desempleados, independientemente de los grupos de edad en los que se sitúen. Resultados similares se han encontrado en Kulik (2000) donde la edad, entre otras variables, se constituye en una determinada actitud como respuesta a la situación de desempleo. Del análisis de las investigaciones sobre el desempleo en la edad adulta, se constata que son cuantitativamente menores que las orientadas hacia otras etapas del desarrollo (Izquierdo, 2008; Izquierdo y Hernández, 2011; Izquierdo, Hernández y Maquilón, 2010).

El género es otra de las variables estudiadas, en relación a la creatividad. En los resultados obtenidos no se aprecian diferencias significativas, en los niveles de creatividad de los participantes, en relación al género. Buendía (1990) indica que el impacto del desempleo puede ser tan negativo en las mujeres como en los hombres. No obstante, la influencia del género es distinta de la que puedan ejercer otras variables.

\section{Conclusiones}

La falta de empleo, las escasas oportunidades, son fenómenos que sólo se reducen a una causa y un adjetivo: falta de creatividad. Respecto a las diferencias en género y creatividad, Baer (2005) apunta que es una cuestión complicada y controvertida. Durante mucho tiempo se ha considerado que existían diferencias de género en creatividad, sobre todo en los niveles más altos, ya que hay más genios y hombres célebres que mujeres en las ciencias, las artes y la tecnología (Baer, 1999; 2005; Eysenck, 1995; Reiss, 1999), aunque las diferencias dependen del campo concreto que se trate, existiendo áreas como la literatura o la danza donde hay bastantes mujeres célebres.

Algunos autores como Eysenck (1995), afirman que el origen de las diferencias en producto creativo entre mujeres y hombres son de tipo biológico, pero los resultados de las investigaciones apuntan a que tales diferencias surgen básicamente de variables ambientales. Autores como Baer (2005) y Eisler y Montuori (2007), plantean que las diferencias de género, en producto creativo, pueden explicarse por una combinación de factores tales como: 1) las diferencias de género en el acceso a la enseñanza y a los recursos, ya que, a lo largo de la historia, los hombres han controlado el acceso de las mujeres a muchos campos, limitando así su desarrollo. Según estudios de Mataud, Rodríguez y Grande (2007), los hombres son más creativos que las mujeres en estudios medios; y la creatividad es igual en hombres universitarios y mujeres universitarias; 2) las diferentes expectativas y la socialización diferencial de mujeres y hombres; 3) el control por parte de los hombres de la norma por la que un determinado logro se considera o no creativo. Además, en los pocos estudios en que se han estudiado las diferencias de género en pensamiento creativo, los resultados han sido contradictorios, encontrándose en algunos que no hay diferencias, en otros que los hombres puntúan más alto, y en otros que son las mujeres las que puntúan más en pensamiento creativo (Baer, 1999).

En cuanto a la edad y su repercusión en la creatividad, podemos decir que existen diferentes estudios (Coriat, 1990) donde se ha comprobado que hacia los 5 años de edad, el porcentaje de niños creativos alcanza el $90 \%$ pero hacia los 7 años sólo encontramos el 10\%, y va disminuyendo alarmantemente hasta llegar al 2\% a los 45 años. En consecuencia, casi todos los seres humanos abordan la vida con un gran potencial de creatividad; potencial que es mermado poco a poco por malos hábitos, por ideas que frenan la espontaneidad y por la exigencia de conductas convencionales. Las personas, en general, pierden creatividad con los años. La mayoría de las personas no piensan que pueden ser creativas, tienen más prejuicios e inhibiciones, hacen menos preguntas, asumen menos riesgos.... El adulto piensa entre tres y seis alternativas para cualquier situación, mientras que el niño piensa en sesenta.

En definitiva, la creatividad tiene mucho que ver con no sentirse limitado por las reglas ni aceptar las restricciones 
impuestas por la sociedad. Es importante, porque es una capacidad innata, que nos ayuda a enfrentarnos a los cambios que se están produciendo en la sociedad. Nos ofrece muchas herramientas para lograr alcanzar nuestras metas en la vida manera exitosa. La creatividad, más que un merecimiento intelectual o habilidad, es una actitud ante la vida. La creatividad es un bien social y personal admirado y temido al mismo tiempo. Cuando hablamos de creatividad, no solo estamos hablando en términos de productividad e invención, sino también de salud física, ya que puede ayudarnos mucho en nuestra senectud. La expresión creativa fomenta sensaciones positivas, que incitan a una perspectiva optimista y a un sentido del bienestar. Y, promoviendo emociones positivas, la creatividad también promueve funciones de inmunidad positivas.

Podemos decir que la creatividad es una forma de ser, pensar y sentir que nos ayuda a trabajar de una forma distinta y que maximiza las posibilidades de producir ideas novedosas y adecuadas en cualquier contexto de actividad humana. Somos creativos fundamentalmente cuando hacemos lo que nos gusta, cuando disfrutamos haciendo algo, aunque requiera un esfuerzo (López y Martín, 2010). Somos creativos por necesidad adaptativa ante los problemas que nos van

\section{Referencias}

Álvaro, J. L. (1992). Desempleo y bienestar psicólogico. Madrid: Siglo XXI.

Baer, J. (1999). Gender differences. En M. A. Runco y S. Pritzker (Eds.) Encyclopedia of Creativity (pp. 753-758). San Diego: Academic Press.

Baer, J. (2005). Gender and creativity. Annual Meeting of the American Psychological Association. Washington: DC.

Banks, M. H. (1992). Desempleo y salud mental: investigaciones británicas recientes. En J. L. Álvaro, J. R. Torregrosa y A. Garrido (Comps.), Influencias sociales y psicológicas en la salud mental (pp. 143-170). Madrid: Siglo XXI.

Buendía, J. (1990). Psicopatología del desempleo. Anales de Psicología, 6 (1), 21-36.

Caputo, R. K., Cianni, M. (2001). Correlates of voluntary vs. involuntary parttime employment among US women. Gender, Work and Organization, 8 (3), 311-325.

Corbalán, F. J., Martínez, F., Donolo, D., Tejerina, M., Limiñana, R. M. (2003). CRE A Inteligencia Creativa. Una medida cognitiva de la creatividad. Madrid: TEA Ediciones.

Corialt, A. R. (1990). Los niños superdotados. Barcelona: Herder

Csikszentmihalyi, M. (1998). Creatividad. El fluir y la psicología del descubrimiento y la invención. Barcelona: Paidós.

Del Pozo, J. A., Ruiz, M. A., Pardo, A. y San Martín, R. (2002). Efectos de la duración del desempleo entre los desempleados. Psicothema, 14 (2), 440-443.

Eisler, R. y Montuori, A. (2007). Creativity, society, and the hidden subtext of gender: A new contextualized approach. World Futures. The Journal General Evolution. 63 (7), 479-499.

EPA: Encuesta de Población Activa (2012). Indicadores de la Encuesta de Población Activa. Instituto Nacional de Estadística. Recuperado el 9 de febrero de 2012, de: http://www.ine.es/jaxi/menu.do?type=pcaxis\&path=/t22/e308_mnu\&file $=$ inebase $\& \mathrm{~N}=\& \mathrm{~L}=0$

Eysenck, H. J. (1995). Genius. The natural bistory of creativity. Cambridge: Cambridge University Press.

García, A. M. y García, M. G. (2008). La influencia de los rasgos psicológicos en las actitudes hacia el empleo. Revista de Psicología del Trabajo y de las Organizaciones, 24 (2), 203-233.

Garrido-Fernández, M. (1995). Desempleo y psicopatología. Un estudio empirico e interpretación psicodinámica. Valencia: Promolibro.

Garrido-Luque, A. (1996). Psicología social del desempleo. En J. L. Alvaro, A. Garrido y J. L. Torregrosa (Coords), Psicología social aplicada (pp. 121-154). Madrid: McGraw-Hill.

Garrido-Luque, A. (2006). Sociopsicología del Trabajo. Barcelona: UOC.

Izquierdo, T. (2008). El desempleo en los mayores de 45 años. Jaén: CES. surgiendo y ante los que las respuestas usuales ya no valen. Cuando no solamente actuamos movidos por motivos externos (trabajo, dinero, reconocimiento), sino por las sensaciones que experimentamos durante la actividad. Somos creativos simplemente creyendo que podemos serlo, conociendo nuestras fortalezas y virtudes, trabajando para desarrollar unas y compensar otras, utilizando estrategias y técnicas de pensamiento que maximicen la posibilidad de ver la realidad, los problemas y los proyectos de otra manera, tolerando la ambigüedad, asumiendo riesgos, siendo persistente en el trabajo, conociendo y expresando nuestras emociones, etc. Entender la creatividad como un fenómeno complejo, supone conceptuarla como consecuencia de múltiples interacciones que la hacen posible y al mismo tiempo condiciona su predicción.

La creatividad es fuente de bienestar y felicidad y puede convertirse en un vehículo para el desarrollo personal y una vía de intervención para la prevención y promoción de salud psicológica. Desde este trabajo se plantea que, en un futuro, se lleven a cabo investigaciones enfocadas a las relaciones entre creatividad, bienestar, felicidad y personas en desempleo.

Izquierdo, T. y Alonso, H. J. (2010). Valores culturales y consecuencias psicosociales del desempleo en América Latina. Revista de Psicología del Trabajo y de las Organizaciones, 26 (2), 123-133.

Izquierdo, T. y Hernández, F. (2011). Orientación profesional y desempleo adulto. En J.J. Maquilón et al. (Coords.), Cambios educativos y formativos para el desarrollo bumano y sostenible (pp. 519-526). Murcia: Editum.

Izquierdo, T., Hernández, F. y Maquilón, J. J. (2010). Psicología del ciclo vital en el desempleo adulto. I Congreso Internacional de Psicología del Trabajo y de las Organizaciones. Buenos Aires, Argentina.

Kulik, L. (2000). The impact of gender and age on attitudes and reactions to unemployed: The Israeli case. Sex Roles, 43, 85-104.

Kulik, L. (2001). Assessing job search intensity and unemployment-related attitudes among young adults: Intergender differences. Journal of Career Assessment, 9 (2), 153-167.

López, O. (2001). Evaluación y desarrollo de la creatividad. Murcia: Servicio de Publicaciones. Universidad de Murcia.

López, O. y Navarro, J. (2008). Estudio comparativo entre medidas de creatividad: TTCT vs. CREA. Anales de Psicologia, 24 (1), 138-142.

López, O. y Martín, R. (2010). Estilos de pensamiento y creatividad. Anales de psicologia, 26 (2), 254-258

Mataud, M.P., Rodríguez, C. y Grande, J. (2007). Gender differences in creative thinking. Personality and individual differences, 43, 1137-1147.

OIT: Organización Internacional del Trabajo (2012). Tendencias mundiales del empleo 2012: Prevenir una crisis mayor del empleo. Recuperado el 21 de febrero de 2012 de: http://www.ilo.org/wcmsp5/groups/public/---dgreports/---dcomm/--publ/ doc uments/ publication/wcms_171699.pdf

Piqueras, R. y Rodríguez, A. (1997). Orientación profesional centrada en las soluciones. Intervención Psicosocial, 6 (3), 317-337.

Reiss, S. M. (1999). Women and creativity. En M. A. Runco y S. Pritzker (Eds.) Encyclopedia of Creativity (pp. 699-708). San Diego: Academic Press.

Sánchez, J. (1994). El bienestar psicológico subjetivo. Escalas de evaluación. Boletín de Psicología, 43, 25-51.

SPEE: Servicio Público de Empleo Estatal (2012). Informes y estudios sobre tendencias del mercado de trabajo. Recuperado el 21 de febrero de 2012, de: http://www.sepe.es/indiceTendencias/indiceTendencias.do?idioma $=\mathrm{es}$

Wanberg, C. R. (1997). Antecedents and outcomes of coping behaviours among unemployed and reemployed individuals. Journal of Applied Psychology, 82 (5), 731-744.

(Artículo recibido: 16-10-2011, revisado: 19-02-2012, aceptado: 23-02-2012) 